\title{
THE ROLE OF SOCIAL MEDIA FOR THE PHYSICIANS AND HOSPITALS TO IMPROVE CONSUMER ENGAGEMENT: A SYSTEMATIC REVIEW
}

\author{
Nadya Aviliyani Taufik, Wahyu Sulistiadi \\ Hospital Administration Program, Faculty of Public of Health, Universitas Indonesia
}

\begin{abstract}
Background: Social media are dynamic and interactive computer-mediated communication tools that have high penetration rates in the general population in high-income and middle-income countries. However, in medicine and health care, a large number of stakeholders such as physicians and hospitals are unaware of social media's relevance, potential applications in their day-to-day activities, as well as the inherent risks and how these may be attenuated and mitigated. This study aimed to review systematically the role of social media for the physicians and hospitals to improve consumer engagement.
\end{abstract}

Subjects and Method: This systematic review was conducted through six steps: (1) Frame a question (based on a theory); (2) Run a search (on PubMed, and ProQuest); (3) Read the abstract and title of the individual papers; (4) Abstract information from the selected set of final articles; (5) Determine the quality of the information in these articles, which was done using a judgment of their internal validity but also using the GRADE criteria; (6) Determine the extent to which these articles were heterogeneous. The dependent variable was consumer engagement. The independent variable was social media for the physicians and hospitals. The keywords for this review were social media, consumer engagement, loyal patient, health information, and health services. The inclusion criteria were review, systematic review, clinical review and guidelines. After review process 6 articles were included in this review.

Results: Hospital marketing strategies through social media vary in all regions of the world and there were differences between developing and developed countries. Social media was an effective strategy for the physicians and hospitals to improve consumer engagement.

Conclusion: Social media is an effective strategy for the physicians and hospitals to improve consumer engagement.

Keywords: social media, consumer engagement, hospital, physicians, health services.

Correspondence:

Nadya Aviliyani Taufik. Hospital Administration Program, Faculty of Public of Health, Universitas Indonesia. Email: nadya.aviliyani@ui.ac.id. Mobile: 081210108333.

BACKGROUND
Nowadays the development of techno-
logy has developed very rapidly, for-
cing us to keep up with the times. In-
cluding in the world of health, hospi-
tals or health facilities as organizations
must be able to adapt to changes. At
present hospitals and clinics must pick
up opportunities by embracing social

media as a means for them to introduce hospitals profiles or clinics to the public. Besides that social media is also used to better educate the public with health information, and get the trust and satisfaction from patients. The main purpose of choosing social media as a means of marketing by health care providers is to provide 
health information, promote existing health services and products including excellent health services become the characteristic of the Hospital or clinic.

Even now questions and answers about health tips can be done through social media. In all of these ways social media can be the main means of engagement between patients and health care provider. If the hospital or clinic can maintain a good relationship with social media users, it is possible to get loyal patients. Research shows that the use of social media by hospitals and physicians contributes to increased hospital website visitors, hospital brand, and patient recruitment for research projects (Zhang, 2018). In 2007 more than 1000 social media were reported with more than 3 billion active users on the internet.

The amount of time per day used to access social media networks using the internet worldwide increases from 90 minutes per day in 2012 to $135 \mathrm{mi}-$ nutes per day in 2017. A 2011 study found that both patients and physicians using online facilities were increasing including websites, social media to look for health information. In 2014 it was reported that $87 \%$ of American adults use the internet and $72 \%$ of internet users reported seeking health information through online media (Campbell, 2016).

According to Wei et al. (2018) among the various existing social media, Facebook which was created in 2004 is the largest social network with 2.07 billion active users every month around the world in 2017, followed by Facebook Messenger, WhatsApp, and YouTube. In the United States, 70\% of hospitals use Facebook. In Western
Europe, several hospitals in the Netherlands and the UK are seen as pioneers in adopting social media in the health sector, and the most widely adopted social media platforms by hospitals are YouTube, LinkedIn, and Facebook. Another case in China, the most popular social media in China is WeChat (67\%).

\section{SUBJECTS AND METHOD \\ 1. Study Design \\ This was systematic review study with the PRISMA method to search for journals. Data were obtain from online database such as ProQuest and Pub- Med, with keywords "Social Media" and "Consumer Engagement" and "Loyal patient" and "health informati- on" and "health services", the researc- her found 2489 documents.}

\section{Inclusion and Exclusion Crite-} ria

The researcher limited the journal paper numbers by duplication and years (2017 to 2018) it end up with 497 articles. The researcher limited again based on the titles, picked 16 articles. However, after further reading including the abstract sections, the researcher got 6 articles.

\begin{tabular}{l}
\hline RESULTS \\
The majority of existing websites have \\
a presence on social media networks, \\
namely Facebook, Twitter, YouTube \\
and Instagram. It is unfortunate if the \\
Hospital website does not use social \\
media as a medium to expand the sco- \\
pe of its services through online media. \\
In Kuwait, only one government hos- \\
pital has a Twitter and Instagram acc- \\
ount with less than 70o followers in \\
each account (Alhuwail, 2018).
\end{tabular}


Whereas in China, only actively using social media in 2017, as many as 197 new hospitals that are best in town started using WeChat (67\%), Sina Weibo (45\%) (local social media) to connect with the community, this shows a large scale and sustainable adoption.

In contrast to Taiwan, based on research in 2017, Facebook is the most widely used social media. According to Taiwan's Institute for Information Industry known Facebook was most popular with $90.9 \%$ of Internet users, followed by LINE (87.1\%), YouTube (60.4\%). From 417 hospitals, 213 (51$.1 \%)$ already have their own Facebook fan pages (Po-Chin Yang, 2018). As with the Spanish state, almost all hospitals in Spain already have a website. Only 5\% do not have a website, and all of them are public hospitals. But 50\% of the best hospitals in Spain do not have Facebook accounts as their social media. 23\% share the channel with the Central Hospital (Hospitals Group) or from the channel in the health

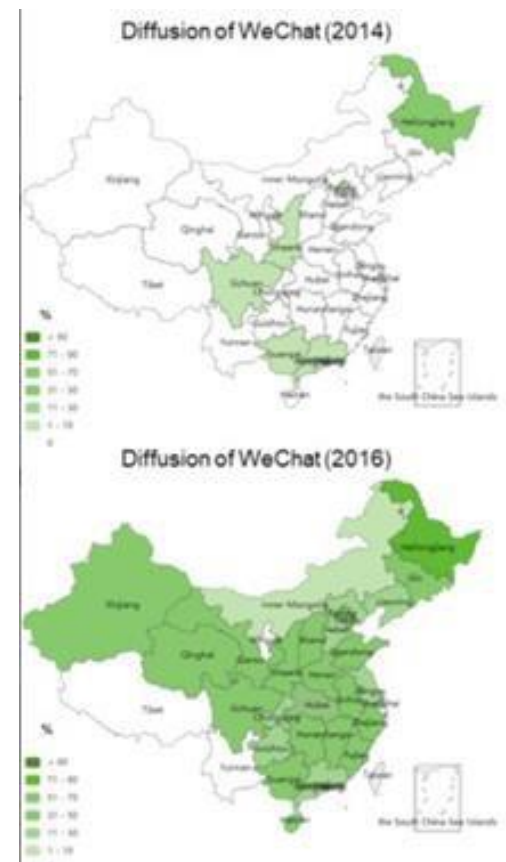

department where the hospital is located. 27\% already have their own Facebook account.

This percentage is almost the same as using a Twitter account, where only $26 \%$ of the best hospitals in Spain have their own Twitter account, 28\% share an account with the Central Hospital and 46\% do not have a social network. Only 20\% use YouTube. Overall Twitter, Facebook and YouTube are the three most social networks in Spain (Costa-Sánchez, 2016). Based on research conducted in U.S., a previous study in 2014 found that 99.41\% (3351/3371) of hospitals had a Facebook page, 1713 (50.82\%) having a Twitter, 3351(99.41\%) having a Foursquare. Also it is known the most frequently used theme is the sharing of health information which is $35.81 \%$ (424/1184) of the post analyzed. The overall picture of the most widely used social media in a country can be seen in Figure 2.

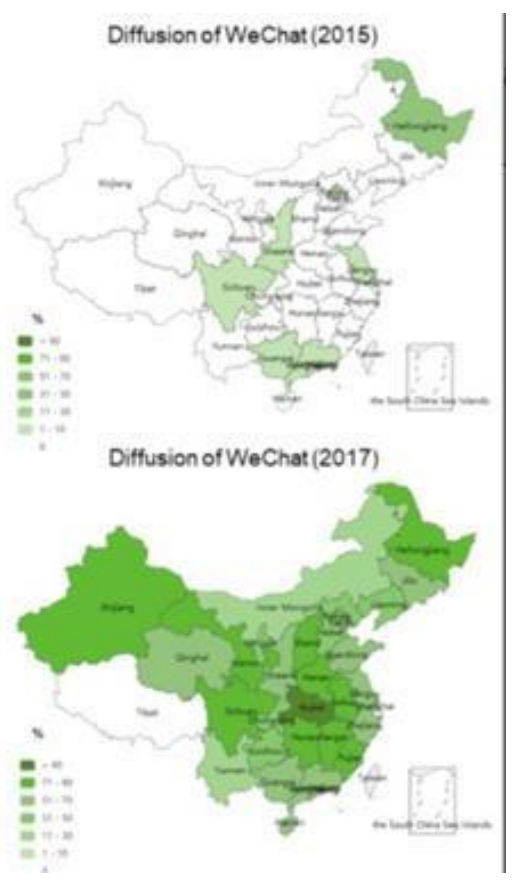

Figure 1. Diffusion of WeChat across the best tertiary hospitals over time (2009-2017) Source: Zhang et al. (2018)

The 5th International Conference on Public Health Best Western Premier Hotel, Solo, Indonesia, February 13-14, 2019 | 550 https://doi.org/10.26911/theicph.2019.04.52 


\section{Figure 2. The Most Social Media Use In a Country}

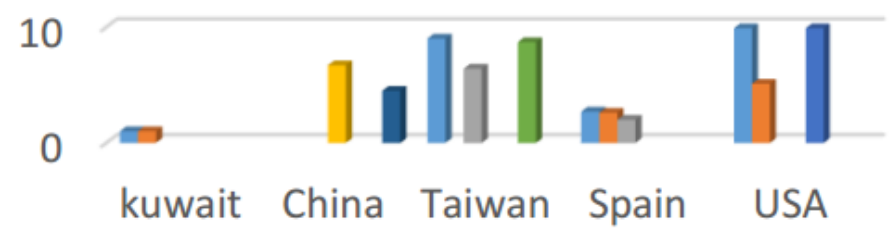
Facebook $\quad$ Twitter
YouTube
WeChat
Foursquare $\square$ Line
Sina Weibo

Figure 2. The Most Social Media Use in a Country

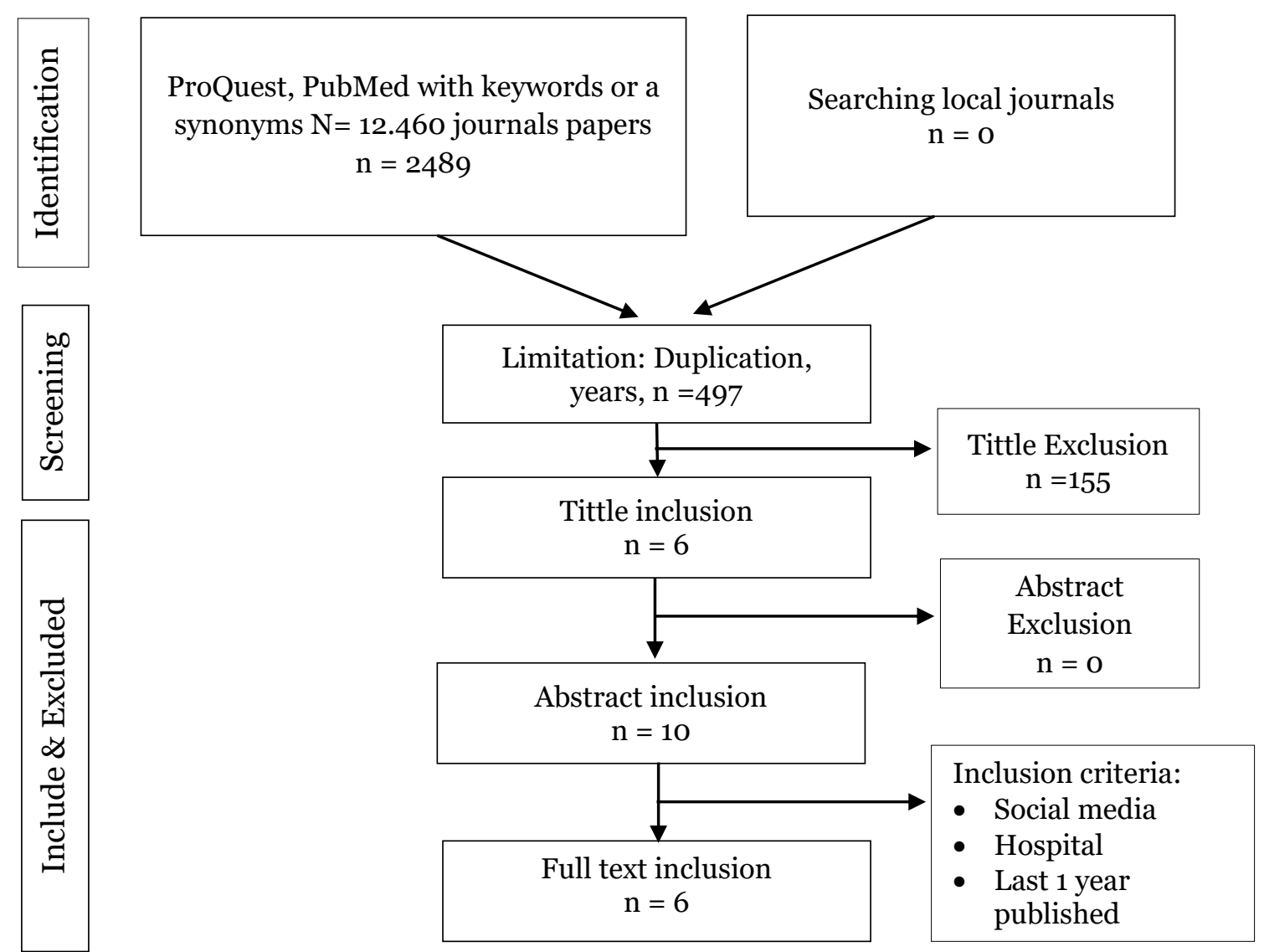

Figure 3. Categories of Social Media Used by Participants

Source: Campbell et al. (2016)

Other findings from the results of the study in US include various benefits and shortcomings felt by doctors in using social media. The 17 doctors who participated in this study had several social media network accounts including Twitter (10), Facebook (7), Pinterest (3), YouTube (2) and Tumblr (2) 
as well as freestanding Blogs (13). 5 of the participants were also active in traditional social media such as radio, newspaper columns and magazines (Figure 3) (Campbell, 2016).

$\frac{\text { DISCUSSION }}{\text { Most of the doctors expressed the opi- }}$
nion that more benefits were felt than the obstacles faced in using social media. The perceived benefits of using social media include supporting careers or supporting their research efforts, as a way to improve themselves from reading other people's posts and reading the literature, increasing their coverage, and providing a place for them to express themselves. While the use of social media from the patient's side is to make it easier for them to gain access to accurate, trusted and easily understood information sources, because social media is very easily accessible in general and can be done anywhere (patients do not have to go to the hospital to find information).

This method is an increasing way of communication and speed in communication while breaking the traditional way of communicating. From the results of various studies it can be seen that the adoption of Hospital marketing techniques through social media varies greatly in all regions of the world. In developing countries it seems that it has begun to adopt marketing techniques through social media by building Hospital websites and profiling hospitals through social networks, although it is still limited to just a few hospitals (new hospitals or best hospitals in the country).

The main factor that causes it is because developing countries are new to "getting to know" social media technology. There are still many hospitals that still use manual systems, even though the Hospital's information system is running. There are still a lot of things needed to develop a hospital information management system including preparing human resources who are ready to accept and be able to adapt to technological sophistication. The culture and the society of a country also influence the absorption of technology. As with developed countries, marketing through social media has long been applied in all health care facilities including people who have updated technology.

The number of hospitals that already know the importance of social media in connecting hospitals to patients has increased and social media has been identified as a general strategy to improve communication between patients and doctors. In addition to the above reasons, social media is also reported to be able to improve communication among Hospital employees, can facilitate networks, can increase the number of visitors to Hospital websites, can build Hospital images, and can be useful as research projects (de Belt, 2012). It can be concluded that Facebook is the social media with the largest users and the most frequently used throughout the world. This is because Facebook's features tend to be attractive and easy to use for almost all ages, easy to access and almost unpaid.

Some things that benefit from using Facebook as a marketing strategy are: (1) very easy and fast in creating a Facebook account, (2) Facebook 
provide analysis to see and read interactions, demographics, and other feedback about the market community that we are targeting, (3) Facebook provides a "voice of the customer." Where communication takes place in two directions, this is added value when service providers and consumers can interact to provide feedback, (4) Facebook expands your reputation and message of branding, (5) Facebook increases search engine visibility and user traffic, (6) Facebook is an advertising platform that provides media with target market, (7) many Facebook users from anywhere who can become prospective patients. For many (though not all) professions, the presence of Facebook presented correctly can prospect patients, new patients, referrals and repeat business (Griffis, 2014).

\section{REFERENCE}

Alhuwail D, AlMeraj Z, Boujarwah F (2018), Evaluating hospital websites in Kuwait to improve consumer engagement and access to health information: a cross sectional analytical study, BMC Medical Informatics and Decision Making. BMC Medical Informatics and Decision Making. 18:82. doi: 10.1186/s12911018o660-4.

Campbell L, Evans Y, Pumper M, Moreno MA (2016). Social media use by physicians: a qualitative study of the new frontier of medicine BMC Medical Informatics and Decision Making. BMC. doi: 10.1186/s12911-016-0327-y.
Costa SC, López MT, Rodríguez JJV (2016). Spanish hospitals in the social web.The management of Facebook and Twitter by Hospital Sant Joan de Déu (Barcelona), Revista Latina de Comunicación Social \# 071 - Pages1.108 to 1.130 doi: $10.4185 /$ RLCS-2016-1137en.ISSN 1138-5820.

de Belt V TH, Berben SA, Samsom M, Engelen LJ, Schoonhoven L (2012). Use of Social Media by Western European Hospitals: Longitudinal. J Med Internet Res. 2012 May 1; 14(3):e61. doi: 10.2196/jmir.1992.Griffis HM, Kilaru AS, Werner RM, Asch DA, Hershey JC, Hill J, Ha YP et al., (2014). Use of Social Media Across US Hospitals: Descriptive Analysis of Adoption and Utilization. J Med Internet Res. 2014 Nov; 16(11): e264. Published online 2014 Nov 27. doi: 10.2196/jmir.3758.

Yang PC, Lee WC, Liu HY, Shih MJ, Chen TJ, Chou LF, Hwang SJ (2018). Use of Facebook by Hospitals in Taiwan: A Nationwide Survey, International Journal of Environmental Research of Public Int $\mathrm{J}$ Environ Res Public Health. 2018 Jun; 15(6): 1188. Published online 2018 Jun 6. doi: 10.3390/ijerph15061188. Zhang W, Deng Z, Evans R, Xiang F, Ye Q, Zeng R (2018). Social Media Landscape of the Tertiary Referral Hospitals in China: Observational Descriptive Study, J Med Internet Res. 9;20(8):e249. doi:10.2196/jmir.9607. 